\title{
Recursion-based Analysis for Information Propagation in Vehicular Ad Hoc Networks
}

\author{
Minming $\mathrm{Ni}^{*}$, Jianping $\mathrm{Pan}^{\dagger}$, Miao Hu*, Zhangdui Zhong* \\ ${ }^{*}$ State Key Lab. of Rail Traffic Control and Safety, Beijing Jiaotong University, Beijing, China \\ ${ }^{\dagger}$ University of Victoria, Victoria, BC, Canada
}

\begin{abstract}
Effective inter-vehicle communication is fundamental to a decentralized traffic information system based on Vehicular Ad Hoc Networks (VANETs). To reflect the uncertainty of the information propagation, most of the existing work was conducted by assuming the inter-vehicle distance follows some specific probability models, e.g., the lognormal or exponential distribution, while reducing the analysis complexity. Aimed at providing more generic results, a recursive modeling framework is proposed for VANETs in this paper when the vehicle spacing can be captured by a general i.i.d. distribution. With the framework, the analytical expressions for a series of commonly discussed metrics are derived respectively, including the mean, variance, probability distribution of the propagation distance, and expectation for the number of vehicles included in a propagation process, when the transmission failures are mainly caused by MAC contentions. Moreover, a discussion is also made for demonstrating the efficiency of the recursive analysis method when the impact of channel fading is also considered. All the analytical results are verified by extensive simulations. We believe that this work is able to potentially reveal a more insightful understanding of information propagation in VANETs by allowing to evaluate the effect of any vehicle headway distributions.
\end{abstract}

Index Terms-Inter-vehicle communications, general vehicle headway distributions, stochastic characteristics

\section{INTRODUCTION}

Due to the potential to disseminate the safety warnings and traffic information for significantly decreasing the number of road accidents, the Vehicular Ad Hoc Networks (VANETs) are widely recognized as one of the few core components for the next-generation Intelligent Transportation Systems (ITS) [1]. To further push forward VANETs' development, the US Federal Communications Commission (FCC) has allocated $75 \mathrm{MHz}$ of Dedicated Short-Range Communications (DSRC) spectrum at $5.9 \mathrm{GHz}$ to be used exclusively for Vehicle-toVehicle (V2V) and Vehicle-to-Road Infrastructure (V2R) communications. Besides, IEEE has also completed the standardization process for IEEE 1609.1, 1609.2, 1609.3, and 1609.4 for the Wireless Access of Vehicle Environments (WAVE), which utilizes IEEE 802.11p to handle the media access control issues uniquely happened in the VANET scenario. Moreover, a considerable amount of VANET-oriented projects have been initiated by governments, automotive industry, highway management authorities, and safety organizations, e.g., the Vehicle Safety Consortium (VSC) in USA, the Car-toCar Communications Consortium (C2C-CC) sponsored by the European Union, and the Advanced Safety Vehicle Program (ASV) in Japan.
Among all the research topics in VANETs, the performance of information propagation in the dynamic network scenario, which is fundamental to ITS, is always treated with a high priority. Generally, the difficulties for the information propagation related studies come from the time varying vehicle mobility, the burst-style data traffic loads, and the extremely complicated radio environments. Regarding all these issues, [2] conducted numerous simulation studies for the information propagation distance. After that, the information propagation issue was further studied in [3] which developed numerical method to recursively calculate the probability of successful propagation. With the similar idea of recursive analysis, [4] studied the feature of cluster size in VANETs, and proposed a time/location-critical framework specifically for the emergency message dissemination. In one of the most recent work [5], the information propagation issue was investigated when the vehicles in the network could be categorized into a number of speed distribution-determined traffic streams. Although such work advanced the understanding for the information propagation process in VANETs, most of it were confined to the condition that the vehicle presence on a road segment follows some specific random processes, e.g., the commonly used homogeneous Poisson point process. These carefully selected probability models can significantly reduce the analysis complexity. However, it is often argued that they are in violation of the realistic vehicle spatial distribution when some factors such as the driver behavior, traffic condition, and road type are considered [6].

Inspired by [7], this paper presents a recursive analytical model for the information propagation process by looking into the physical meaning of the expected propagation distance and incorporating the possible factors for a transmission failure, when the vehicle distance headway can be described by a general probability distribution. Considering its generality, this study would offer more insights and enable a more robust design of VANETs-based ITS by allowing for analytical verification of various different headway distributions on successful information propagation. The remainder of this paper is organized as follows. The system model for our recursive analysis is given in Section II. After that, the information propagation distance's stochastic characteristics are derived in Section III. The verifications of our analytical results are shown in Section IV. Moreover, a discussion is also made in Section V to briefly describe how the recursive model could be used when the impact of channel fading is considered. Finally, Section VI concludes this paper. 


\section{SySTEM MODEL}

In our analysis, the random distance $H$ between direct neighboring vehicles, which is termed as the headway distance, follows a general distribution whose density function is denoted as $f_{H}(x)$ with expectation $\mu_{H}$ and variance $\sigma_{H}^{2}$. Without considering the impact of specific routing or transmission schemes on information propagation in VANETs, this paper focuses on the case that each vehicle only attempts to relay the received information via its direct neighbor. If the trial failes after a certain retry limit or without retry due to broadcast, the entire transmission process is terminated, and the total propagation distance $D$ equals the distance from the last receiving vehicle to the origin of the information. Due to the space limit, in this work, we mainly consider the case that the transmission failures are only caused by the MAC contentions. However, based on the proposed recursive analytical framework, more general cases can also be investigated. To demonstrate the framework's effectiveness, a discussion section is arranged at the end of this paper, which includes some brief results for the situation when the channel fading becomes the major cause for the interruption of an information propagation process. A comprehensive version of the more general analyses will be finished as a follow-on work for this paper in the near future.

When the MAC contentions are the only concerns, it is common to assume that each vehicle has the identical transmission range $L$. With this constant transmission range model, a communication can be initiated if the distance between two vehicles is less than $L$. However, due to the design of the backoff algorithm in MAC schemes, it is possible that more than one vehicle may attempt to utilize the channel at the same time, which will lead to a transmission collision if within an interference range. This is common when the network density or traffic load is high at some specific time or locations. There is a lot of existing work focusing on the reliability of VANETs MAC, e.g., the packet reception rate in the IEEE 802.11based VANETs was derived in [8], and the analytical model was further improved in [9] for more accurately describing the Frozen Period(s) in a channel contention process. For this paper, the probability for a successful reception when the MAC contentions are the only concerns is denoted as $p_{\mathrm{s}}$, and treated as a known parameter. Moreover, once the analytical expressions for the characteristics of the information propagation are obtained, $p_{\mathrm{s}}$ could be replaced by the already derived closed-form or empirical expressions, which allow us to reveal a more insightful understanding about the relationship between the system parameters and the comprehensive network performance metrics.

\section{ThEORETICAL ANALYSIS}

According to the system model, it is clear that the information propagation is a typical renewal process. In other words, if the expectation of the propagation distance $D$ can be obtained as $\mu_{D}$, then each vehicle should have the same potential to further propagate the information forward with expectation $\mu_{D}$. Interestingly, this interpretation of the propagation distance's expectation allows us to look into the characteristics of $D$ from a recursive aspect. With the headway distribution, the probability for the first receiving vehicle to be located at distance $\tau$ to a tagged transmitting vehicle is $f_{H}(\tau) \mathrm{d} \tau$. For any receiving vehicle, it has probability $p_{\mathrm{s}}$ to successfully finish the information reception, and has the potential to further relay the information forward for an expected distance $\mu_{D}$. Hence, we could have the following recursion:

$$
\mu_{D}=\int_{0}^{L}\left(\tau+\mu_{D}\right) f_{H}(\tau) p_{\mathrm{s}} \mathrm{d} \tau .
$$

Based on (1), it is easy to obtain the following theorem.

Theorem 1. When the successful transmission probability determined by the MAC contention can be presented by $p_{\mathrm{s}}$, the expected information propagation distance is given by

$$
\mu_{D}=\frac{p_{\mathrm{s}} \int_{0}^{L} \tau f_{H}(\tau) \mathrm{d} \tau}{1-p_{\mathrm{s}} F_{H}(L)},
$$

where $F_{H}(\cdot)$ is the cumulative distribution function $(C D F)$ of the headway distance $H$.

The proof of Theorem 1 is quite straight-forward, which is ignored here. An obvious but also interesting observation of Theorem 1 is that, when the successful transmission probability could be treated as independent of the transmission distance, the expected information propagation distance is irrelevant to the probability distribution of vehicle headway beyond the transmission range $L$. As will be seen later, the variance of the propagation distance under this situation is not affected by the headway distribution beyond $L$ either. This feature would be useful when we are trying to acquire a headway distribution from a tremendous amount of field test data, and further using it for calculating the stochastic characteristic of the information propagation distance.

It is clear that there still exists an integral in the numerator of (2), which might not be easy to be calculated when $f_{H}(\cdot)$ is involved with some complicated functions. To reduce the reliance on numerical calculations, some attempts can be made to develop a bound estimation of the expected propagation distance.

Corollary 1. The bound of the expected information propagation distance could be given as

$$
\begin{gathered}
\frac{p_{\mathrm{s}} \mu_{H}}{1-p_{\mathrm{s}} F_{H}(L)}-\frac{\sqrt{\sigma_{H}^{2}+\left(L-\mu_{H}\right)^{2}}-\left(L-\mu_{H}\right)}{2\left(1-p_{\mathrm{s}} F_{H}(L)\right)} \\
\leq \mu_{D} \leq \frac{p_{\mathrm{s}} \mu_{H}-p_{\mathrm{s}} L+p_{\mathrm{s}} L F_{H}(L)}{1-p_{\mathrm{s}} F_{H}(L)} .
\end{gathered}
$$

Proof: The left part of the inequality is based on the results in [10]: for any random variable $Z$ with $\operatorname{PDF} f(z)$, mean $\mu$, and finite variance $\sigma^{2}$, the following holds

$$
\int_{z}^{\infty} t f(t) \mathrm{d} t \leq \frac{\sqrt{\sigma^{2}+(z-\mu)^{2}}+(z-\mu)}{2} .
$$

Hence,

$$
\begin{aligned}
\int_{0}^{L} \tau f_{H}(\tau) \mathrm{d} \tau & =\int_{0}^{\infty} \tau f_{H}(\tau) \mathrm{d} \tau-\int_{L}^{\infty} \tau f_{H}(\tau) \mathrm{d} \tau \\
& \geq \mu_{H}-\frac{\sqrt{\sigma_{H}^{2}+\left(L-\mu_{H}\right)^{2}}-\left(L-\mu_{H}\right)}{2}
\end{aligned}
$$


Besides, from (5) it is also clear that

$$
\begin{aligned}
\int_{0}^{L} \tau f_{H}(\tau) \mathrm{d} \tau & \leq \int_{0}^{\infty} \tau f_{H}(\tau) \mathrm{d} \tau-L \int_{L}^{\infty} f_{H}(\tau) \mathrm{d} \tau \\
& =\mu_{H}-L\left(1-F_{H}(L)\right) .
\end{aligned}
$$

By combining the results of (6) and (7), the bounds of $\mu_{D}$ could be obtained.

The advantage of having such bounds relies on the fact that, with an arbitrary headway distribution, the expected propagation distance could be easily estimated by the mean and variance of the vehicle headway, both of which can be easily obtained with field data. This will be very helpful, if some rough and quick estimations are needed.

Meanwhile, the variance $\sigma_{D}^{2}$ of the successful propagation distance is measured as follows:

Theorem 2. Given the successful transmission probability $p_{\mathbf{s}}$, the variance of information propagation distance $D$ can be calculated by

$$
\sigma_{D}^{2}=\frac{p_{\mathrm{s}} \int_{0}^{L} \tau^{2} f_{H}(\tau) \mathrm{d} \tau}{1-p_{\mathrm{s}} F_{H}(L)}+\mu_{D}^{2} p_{\mathrm{s}} \frac{1-F_{H}(L)}{1-p_{\mathrm{s}} F_{H}(L)} .
$$

Proof: According to the variance decomposition formula (a.k.a. the Eve's Rule), the recursion for $\sigma_{D}$ can be given as below conditional on the distance between the first transmitter and receiver is $\tau$

$$
\sigma_{D}^{2}=\mathbf{V}(D)=\mathbf{V}[\mathbf{E}(D \mid \tau)]+\mathbf{E}[\mathbf{V}(D \mid \tau)] \quad .
$$

According to the mathematical definition of a random variable's variance $\mathbf{V}(X)=\mathbf{E}\left[\left(X-\mu_{X}\right)^{2}\right]$, the first part in (9] can be expanded based on its actual physical meaning as

$$
\begin{aligned}
\mathbf{V}[\mathbf{E}(D \mid \tau)]= & \int_{0}^{\infty}(\mathbf{E}[\mathbf{E}(D \mid \tau)-\mathbf{E}(D)])^{2} f_{H}(\tau) p_{\mathbf{s}} \mathrm{d} \tau \\
= & \int_{0}^{L}\left(\tau+\mu_{D}-\mu_{D}\right)^{2} f_{H}(\tau) p_{\mathbf{s}} \mathrm{d} \tau \\
& +\int_{L}^{\infty}\left(0-\mu_{D}\right)^{2} f_{H}(\tau) p_{\mathbf{s}} \mathrm{d} \tau \\
= & p_{\mathbf{s}}\left(\int_{0}^{L} \tau^{2} f_{H}(\tau) \mathrm{d} \tau+\mu_{D}-\mu_{D}^{2} F_{H}(L)\right)
\end{aligned}
$$

Similarly,

$$
\mathbf{E}[\mathbf{V}(D \mid \tau)]=\int_{0}^{L} \mathbf{V}(D) f_{H}(\tau) p_{\mathbf{s}} \mathrm{d} \tau=p_{\mathbf{s}} \mathbf{V}(D) F_{H}(L)
$$

Therefore, the recursion in (9) can be simplified to

$$
\sigma_{D}^{2}=p_{\mathbf{s}}\left(\int_{0}^{L} \tau^{2} f_{H}(\tau) \mathrm{d} \tau+\mu_{D}-\mu_{D}^{2} F_{H}(L)+\sigma_{D}^{2} F_{H}(L)\right) .
$$

Finally, $\sigma_{D}^{2}$ can be directly presented as shown in (8), hence Theorem 2 is proved.

With the similar method we used for deriving $\mu_{D}$ 's bounds, the following corollary could be obtained.

Corollary 2. Given $p_{\mathrm{s}}$ and the vehicle headway distribution, the variance of the information propagation distance can be estimated as

$$
\begin{aligned}
\mu_{D}^{2} p_{\mathrm{s}} \frac{1-F_{H}(L)}{1-p_{\mathrm{s}} F_{H}(L)} \leq \sigma_{D}^{2} \leq & \frac{p_{\mathrm{s}} L \mu_{H}-p_{\mathrm{s}} L^{2}\left(1-F_{H}(L)\right)}{1-p_{\mathrm{s}} F_{H}(L)} \\
& +\mu_{D}^{2} p_{\mathrm{s}} \frac{1-F_{H}(L)}{1-p_{\mathrm{s}} F_{H}(L)}
\end{aligned}
$$

Proof: First, the inequality shown below is easy to be obtained.

$$
\begin{aligned}
\int_{0}^{L} \tau^{2} f_{H}(\tau) \mathrm{d} \tau & \leq L \int_{0}^{L} \tau f_{H}(\tau) \mathrm{d} \tau \\
& =L \mu_{H}-L \int_{L}^{\infty} \tau f_{H}(\tau) \mathrm{d} \tau \\
& \leq L \mu_{H}-L^{2}\left(1-F_{H}(L)\right)
\end{aligned}
$$

Therefore, by replacing the integral $\int_{0}^{L} \tau^{2} f_{H}(\tau) \mathrm{d} \tau$ in (8), the right side of (13) is proved. The left side of (13) is obvious according to (8). Hence, the Corollary 2 is proved.

Besides the expectation and standard deviation of the information propagation distance, the number $N$ of vehicles included in an information propagation process, which is often termed as the Cluster Size in the VANETs literature, can also be derived by the recursive analysis. The recursion for the expectation of $N$ could be written as follows

$$
\mu_{N}=\int_{0}^{L}\left(1+\mu_{N}\right) f_{H}(\tau) p_{\mathrm{s}} \mathrm{d} \tau
$$

and another new theorem can be obtained.

Theorem 3. The expected number of vehicles included in a single information propagation process can be calculated by the successful reception probability and the headway distribution as

$$
\mu_{N}=\frac{p_{\mathrm{s}} F_{H}(L)}{1-p_{\mathrm{s}} F_{H}(L)} .
$$

Moreover, the recursive method can also be used to derive the probability distribution of $D$, as

Theorem 4. Denote function $F_{D}(s)$ as the CDF of the information propagation distance $D$, it can be recursively calculated as

$$
F_{D}(s)=\left\{\begin{aligned}
1-p_{\mathrm{s}} F_{H}(L), & s=0 \\
1-p_{\mathrm{s}} F_{H}(L)-\left(1+p_{\mathrm{s}}\right) F_{H}(s) & \\
\quad+\int_{0}^{s} f_{H}(\tau) F_{D}(s-\tau) \mathrm{d} \tau & 0<s \leq L \\
1-F_{H}(L)+\int_{0}^{L} f_{H}(\tau) F_{D}(s-\tau) \mathrm{d} \tau, & s>L
\end{aligned}\right.
$$

Proof: The three cases listed in the piecewise-function need to be discussed separately. When $s=0$, it is easy to see that

$$
\begin{aligned}
F_{D}(0) & =\operatorname{Pr}\{D<0\}=1-\operatorname{Pr}\left\{\text { the } 1^{\text {st }} \text { trans. is successful }\right\} \\
& =1-F_{H}(L) p_{\mathrm{s}} .
\end{aligned}
$$

For the case $0<s \leq L, F_{D}(s)=1-\operatorname{Pr}\{D>s\}$. As shown in Fig. 1, if the distance between the origin and the first receiver is within the range $[s, L]$, the information propagation distance will always be longer than $s$; however, if the first receiver is located at location $\tau \in[0, s]$, then the information has to be 


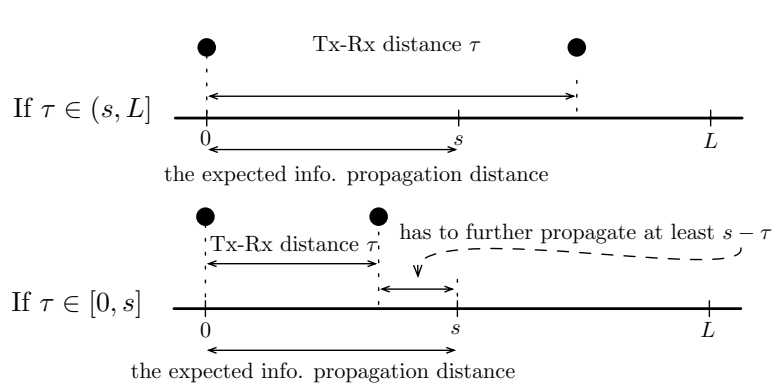

Fig. 1. Calculation of information propagation distance's distribution

TABLE I

BASIC MAC PARAMETERS

\begin{tabular}{c|c||c|c}
\hline Bit Rate $\left(R_{\mathrm{b}}\right)$ & $11 \mathrm{Mbps}$ & Propa. Delay $(\delta)$ & $2 \mu \mathrm{s}$ \\
\hline Slot Time $(\sigma)$ & $20 \mu \mathrm{s}$ & $D I F S$ & $50 \mu \mathrm{s}$ \\
\hline MAC Header & 224 bits & $W_{0}$ & 32 \\
\hline PHY Header & 192 bits & $E\left[L_{\mathrm{P}}\right]$ & 8000 bits \\
\hline
\end{tabular}

further propagated at least distance $s-\tau$ to make sure $D>s$. Therefore,

$$
\begin{aligned}
F_{D}(s)= & 1-\operatorname{Pr}\{D>s\} \\
= & 1-\left(p_{\mathbf{s}}\left(F_{H}(L)-F_{H}(s)\right)+\int_{0}^{s} f_{H}(\tau) \overline{F_{D}}(s-\tau) \mathrm{d} \tau\right) \\
= & 1-p_{\mathbf{s}} F_{H}(L)-\left(1+p_{\mathbf{s}}\right) F_{H}(s) \\
& +\int_{0}^{s} f_{H}(\tau) F_{D}(s-\tau) \mathrm{d} \tau
\end{aligned}
$$

where $\overline{F_{D}}(\cdot)$ is the complementary cumulative distribution function (CCDF) of the propagation distance $D$. Finally, when $s>L$, the first vehicle in the information propagation process should be located within the origin's transmission range $L$, and should relay the information with distance at least $s-L$, whose probability is $\overline{F_{D}}(s-L)$, hence,

$$
F_{D}(s)=1-\int_{0}^{L} f_{H}(\tau) \overline{F_{D}}(s-L) \mathrm{d} \tau .
$$

By combining all the above three parts of results, Theorem 4 is proved.

\section{EVALUATION}

To illustrate the effect generated by the MAC contentions, the analytical results we obtained in [9] are directly applied here to calculate the average transmission successful probability $p_{\mathrm{s}}$. In the simulation, the constant transmission range $L$ for each vehicle is set to $100 \mathrm{~m}$, and all the vehicles are randomly distributed along a road segment according to a Poisson process with density $\lambda$, which means that the vehicle headway should follow an exponential distribution with mean $1 / \lambda$ and variance $1 / \lambda^{2}$. To demonstrate the impact of MAC parameters on information propagation, the length $Q$ of the transmission queue in each vehicle is changed from 1 to 3 , and the normalized traffic loads, which is defined as $\beta L_{\mathrm{P}} / R_{\sharp}$, varies from 0 to 0.5 . All the other basic parameters for the IEEE 802.11-based MAC scheme are listed in Table 【.

\footnotetext{
${ }^{1}$ According to the definitions in [9], $\beta$ is the packet arrival rate at each vehicle, $L_{\mathrm{P}}$ is the average packet length, and $R_{\mathrm{b}}$ is the bit rate.
}

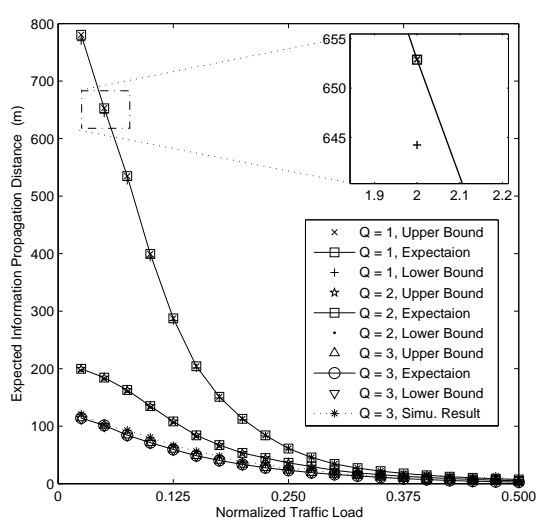

Fig. 2. Expected Information Propagation Distance vs. Normalized Traffic Load, while $\lambda=1 / 5$ (vehicle per meter)

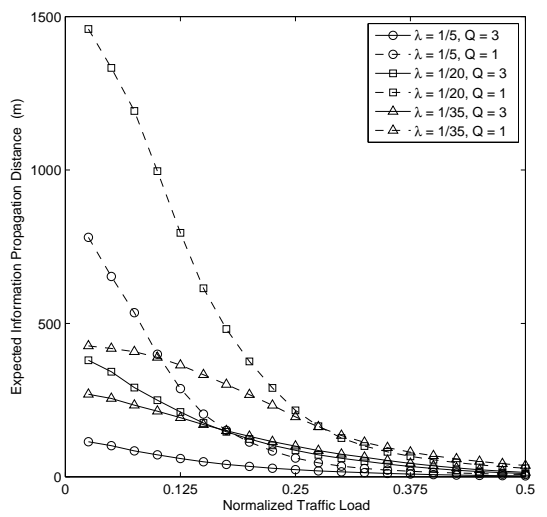

Fig. 3. Expected Information Propagation Distance vs. Normalized Traffic Load, with changed $\lambda$

In Fig. 2, both the analytical and simulation results for the expected information propagation distance are demonstrated with varying normalized traffic loads and queuing lengths at each vehicle, while the expectation of the headway distribution is fixed at 5 meters. As shown in the figure, the analytical results match the simulation results. With the analyses in [9], the successful reception rate $p_{\mathrm{s}}$ is expected to decrease with the increased traffic load, which is mainly due to the significantly increased channel contentions. Moreover, it is also known that a longer transmission queue also reduces the average successful reception probability. This can be explained as that a longer queue can buffer more packets for later transmission, therefore, the probability that a transmission queue is empty is reduced, which indicates that network nodes are more likely to stay in the backoff stage rather than the idle states. Represented in the figure, the expected information propagation distance is decreased with the increased traffic load and queuing length. Besides, the upper and lower bounds estimated from Corollary 1 for different network scenarios are also illustrated in Fig. 2 . It is clear that the bounds are quite tight comparing with the actually calculated results, which will be very useful when the headway distribution is complicated and difficult to be handled with numerical calculations.

In Fig. 3, the expected information propagation distance are illustrated with different traffic load and varied vehicular distribution density $\lambda$. It is obvious that, when $\lambda$ is decreased from $1 / 5$ to $1 / 20$ vehicles per meter, which results in an increase of the expected inter-vehicle distance, the expectation of the information propagation is significantly increased. This 


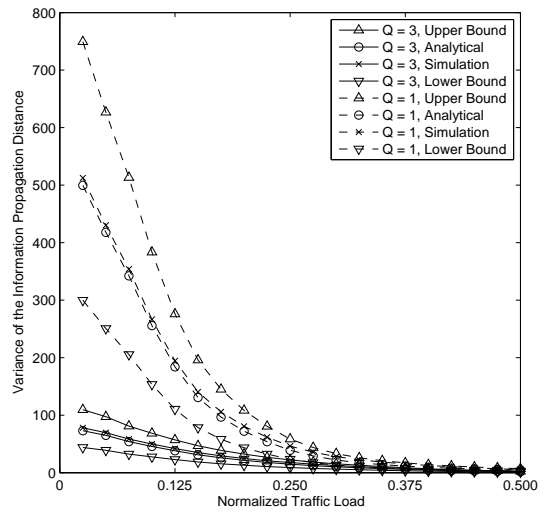

Fig. 4. Variance of the Information Propagation Distance vs. Normalized Traffic Load, while $\lambda=1 / 5$ (vehicle per meter)

is easy to be explained as that, when the hop by hop communication can be carried out successfully with a high probability, increasing the per hop transmission distance will directly increase the total propagation distance. However, when $\lambda$ is further decreased, the expectation of the propagation distance is decreased. This is due to the fact that, with a longer headway expectation, the probability for a transmission failure is also increased, which might start to dominate the overall performance of the information propagation process. The study of the turning point of $\lambda$ 's impact on the expected propagation distance is an interesting topic, and we will dig into it in the near future. It is worth to mention that, to avoid too much overlapping between different data sets, only the results for $Q=1$ and 3 are illustrated in the figure. The changing pattern for $Q=2$ is similar to the ones described above.

The analytical and simulation results for the variance $\sigma_{D}^{2}$ of the information propagation distance for different network scenarios are presented in Fig. 4, with the upper and lower bound estimations obtained by Corollary 2 It is clear that the analytical results and simulation results match with each other, which validates the correctness of the newly proposed recursive analysis method. However, comparing with the tightness between $\mu_{D}$ and its upper or lower bounds demonstrated in Fig. 2. $\sigma_{D}$ 's bound estimations are relatively loose. This is mainly due to the simple mathematical relaxing techniques applied in (14), which should be replaced by more sophisticated inequalities for better tightness. This will be another follow-on work for this paper.

In Fig. 5 and Fig. 6, the expected cluster size and the cumulative probability distribution are illustrated with different network parameters, respectively. It is obvious that the analytical results fit the simulation ones well. The changing pattern of the expected cluster size is identical to the one of the expected propagation distance, which is reasonable due to its physical meanings. For the CDF of the propagation distance, it is clear that when the successful transmission probability $p_{\mathrm{s}}$ is high, which was presented as a shorter queuing length $Q$ in the figure, the corresponding $\mathrm{CDF}$ goes slower to 1 , which means $\mu_{D}$ will be increased. This is also coincident with the results demonstrated in Fig. 2.

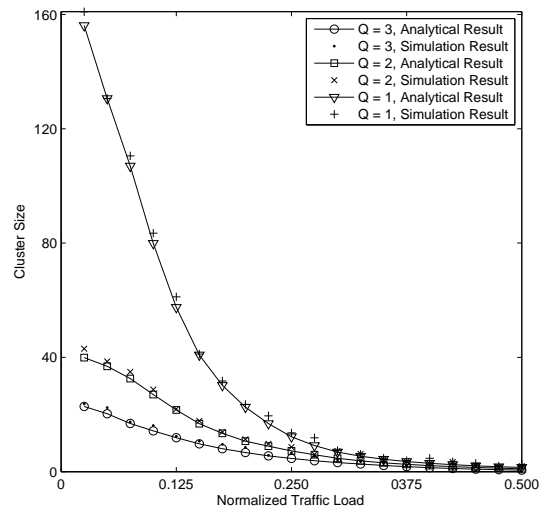

Fig. 5. Expected Cluster Size vs. Normalized Traffic Load, while $\lambda=1 / 5$ (vehicle per meter)

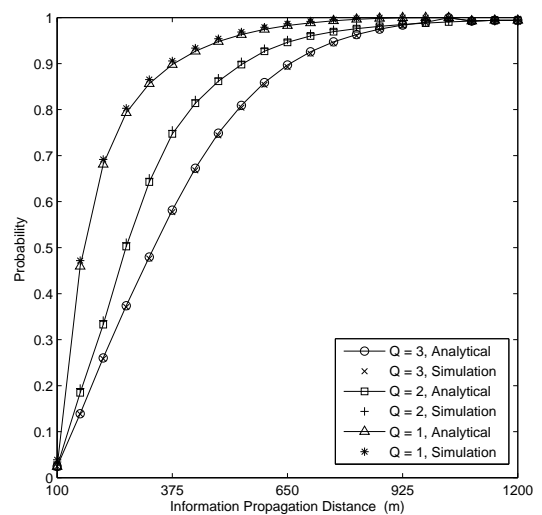

Fig. 6. Cumulative Distribution of Information Propagation Distance vs. Normalized Traffic Load, while $\lambda=1 / 5$ (vehicle per meter)

\section{DisCUSSION}

When the impact of channel fading is considered, the transmission range for each vehicle will be changed from a constant to a random variable. However, with the recursion model described in this paper, similar analyses can also be carried out, which will be breifly discussed in this section. With a specific channel model, given the transmission distance $\tau$, the received signal power can always be described by a conditional probability distribution $f_{P \mid H}(t \mid \tau)$. For example, if we assume that the channel fading follows the Rayleigh model, the reception power's conditional PDF can be presented as

$$
f_{P \mid \tau}(t \mid \tau)=\frac{1}{P_{\mathrm{t}} K\left(d_{0} / \tau\right)^{\alpha}} \exp \left(-\frac{t}{P_{\mathrm{t}} K\left(d_{0} / \tau\right)^{\alpha}}\right), t>0 .
$$

where $P_{\mathrm{t}}$ is the transmission power, $K$ is a constant determined by the hardware features of the transceivers, $\alpha$ is the pathloss exponent, and $d_{0}$ is the reference distance for the far-zone field. To reduce the complexity, here the condition for a successful data transmission is simply set to that the received signal power should be at least higher than the system-determined minimum power threshold $P_{\mathrm{th}}$. However, the case when a successful reception requires the Signal-toInterference-Ratio (SIR) to be higher than a threshold can also be investigated in a similar way. With this model, the successful reception probability needs to be revised to a 
transmission distance $\tau$ determined random variable as

$$
p_{\mathbf{s}}(\tau)=\int_{P_{\mathrm{th}}}^{\infty} f_{P \mid H}(t \mid \tau) \mathrm{d} t
$$

Then the expectation and variance of the information propagation distance could be obtained with the following two theorems, respectively.

Theorem 5. When the impact of channel fading is considered, the expected information propagation distance $\mu_{D}^{\prime}$ is

$$
\mu_{D}^{\prime}=\frac{\mu_{H}-\int_{0}^{\infty} \int_{0}^{P_{\mathrm{th}}} \tau f_{P, H}(t, \tau) \mathrm{d} t \mathrm{~d} \tau}{F_{P}\left(P_{\mathrm{th}}\right)},
$$

where $f_{P, H}(t, \tau)$ is the joint probability distribution function of the received signal power and the transmission distance, and $F_{P}(\cdot)$ is the marginal CDF of the received signal power.

Proof: The recursion of the expected information propagation distance can be written as

$$
\mu_{D}^{\prime}=\int_{0}^{\infty}\left(\tau+\mu_{D}^{\prime}\right) f_{H}(\tau) \int_{P_{\mathrm{th}}}^{\infty} f_{P \mid H}(t \mid \tau) \mathrm{d} t \mathrm{~d} \tau .
$$

With some simple manipulations, we have

$$
\mu_{D}^{\prime}=\frac{\mu_{H}-\int_{0}^{\infty} \tau f_{H}(\tau) \int_{0}^{P_{\mathrm{th}}} f_{P \mid H}(t \mid \tau) \mathrm{d} t \mathrm{~d} \tau}{\int_{0}^{\infty} f_{H}(\tau) \int_{0}^{P_{\mathrm{th}}} f_{P \mid H}(t \mid \tau) \mathrm{d} t \mathrm{~d} \tau} .
$$

According to the property of the conditional probability density function, it is clear that

$$
\int_{0}^{\infty} \tau f_{H}(\tau) \int_{0}^{P_{\mathrm{th}}} f_{P \mid H}(t \mid \tau) \mathrm{d} t \mathrm{~d} \tau=\int_{0}^{P_{\mathrm{th}}} f_{P}(t) \mathrm{d} t=F_{P}\left(P_{\mathrm{th}}\right),
$$

where

$$
f_{P}(t)=\int_{0}^{\infty} f_{P \mid H}(t \mid \tau) f_{H}(\tau) \mathrm{d} \tau
$$

is the PDF of the received signal power when the transmission distance is not given, and $F_{P}(\cdot)$ is the related CDF. By combining all the above results, (22) in Theorem 5 is proved.

Theorem 6. When the impact of channel fading is considered, the variance of the information propagation distance can be calculated by

$$
\sigma_{D}^{\prime 2}=\frac{\int_{0}^{\infty} \tau^{2} f_{H}(\tau) \mathrm{d} \tau-\int_{0}^{\infty} \int_{0}^{P_{\mathrm{th}}} \tau^{2} f_{P, H}(t, \tau) \mathrm{d} t \mathrm{~d} \tau}{F_{P}\left(P_{\mathrm{th}}\right)} .
$$

Proof: Similarly, with the Eve's Rule, and the interpretation for the physical meaning of $\mathbf{V}[\mathbf{E}[D \mid \tau]]$ and $\mathbf{E}[\mathbf{V}[D \mid \tau]]$, we could have

$$
\begin{aligned}
\mathbf{V}[\mathbf{E}[D \mid \tau]] & =\int_{0}^{\infty}\left(\mu_{D}^{\prime}+\tau-\mu_{D}^{\prime}\right)^{2} f_{H}(\tau) \int_{P_{\mathrm{th}}}^{\infty} f_{P \mid H}(t \mid \tau) \mathrm{d} t \mathrm{~d} \tau \\
& =\int_{0}^{\infty} \tau^{2} f_{H}(\tau) \mathrm{d} \tau-\int_{0}^{\infty} \int_{0}^{P_{\mathrm{th}}} \tau^{2} f_{P, H}(t, \tau) \mathrm{d} t \mathrm{~d} \tau,
\end{aligned}
$$

and

$\mathbf{E}[\mathbf{V}[D \mid \tau]]=\sigma_{D}^{\prime 2}\left(\int_{0}^{\infty} f_{H}(t) \mathrm{d} \tau-\int_{0}^{\infty} f_{H}(t) f_{P \mid H}(t \mid \tau) \mathrm{d} t \mathrm{~d} \tau\right)$.

By subsitituting the above two parts into (9), Theorem 6 is proved.

\section{CONCLUSIONS}

In this work, the stochastic characteristics of the information propagation distance were studied with a general headway distribution by a recursion model. Although this paper was focused on the scenario when the MAC contentions are the major causes for a transmission failure, a discussion was also given to demonstrate the recursion model's adaptability when the impact of channel fading is considered. As mentioned in the paper, a series of follow-on work for the recursion model-based analysis will be conducted, including: 1) better bound estimations for propagation distance's variance; 2) more detailed analysis when the SIR becomes the evaluation metric for a successful reception. Moreover, we are also considering to further develop the recursion model for describing the unique store-carry-forward transmission pattern of VANETs, which should be another interesting topic.

\section{ACKNOWLEDGMENT}

This work is partly supported by the NSFC (Grant No. 61401016), State Key Laboratory of Rail Traffic Control and Safety (Grant No. RCS2014ZT33, RCS2014ZQ003), and the Fundamental Research Funds for the Central Universities (Grant No. 2014JBM154).

\section{REFERENCES}

[1] L. Wang, H. Wu, W. Wang, and K.-C. Chen, "Socially enabled wireless networks: resource allocation via bipartite graph matching," IEEE Commun. Mag., vol. 53, no. 10, pp. 128-135, 2015.

[2] X. Yang, Assessment of a self-organizing distributed traffic information system: modeling and simulation. Ph.D. Thesis, University of California, Irvine, 2003.

[3] W. Jin and W. Recker, "Instantaneous information propagation in a traffic stream though inter-vehicle communication," Transportation Research Part B: Methodological, vol. 40, no. 3, pp. 230-250, 2006.

[4] Y. Zhuang, J. Pan, Y. Luo, and L. Cai, "Time and location-critical emergency message dissemination for vehicular ad-hoc networks," IEEE J. Sel. Areas Commun., vol. 29, no. 1, pp. 187-196, 2011.

[5] Z. Zhang, G. Mao, and B. Anderson, "Stochastic Characterization of Information Propagation Process in Vehicular Ad hoc Networks," IEEE Trans. Intell. Transp. Syst., vol. 15, no. 1, pp. 122-135, 2014.

[6] Q. Meng and H. L. Khoo, "Self-similar characteristics of vehicle arrival pattern on highways," Journal of Transportation Engineering, vol. 135, no. 11, pp. 864-872, 2009.

[7] B. X. Wang, T. M. Adams, W. Jin, and Q. Meng, "The process of information propagation in a traffic stream with a general vehicle headway: A revisit," Transportation Research Part C: Emerging Technologies, vol. 18, no. 3, pp. 367-375, 2010.

[8] X. Ma, J. Zhang, and T. Wu, "Reliability Analysis of One-Hop SafetyCritical Broadcast Services in VANETs," IEEE Trans. Veh. Technol., vol. 60, no. 8, pp. 3933-3946, 2011.

[9] M. Ni, Z. Zhong, J. Pan, D. Zhao, and R. Chen, "Non-saturated performance analysis of IEEE 802.11 broadcast in 2-D mobile ad hoc networks," in Proc. of IEEE GLOBECOM, 2012.

[10] G. Gallego, "A minmax distribution free procedure for the $(\mathrm{Q}, \mathrm{R})$ inventory model," Oper. Res. Lett., vol. 11, pp. 55-60, 1992. 